\title{
Ultrasound for malrotation and volvulus: has the time come?
}

\author{
Peter J. Strouse ${ }^{1}$
}

Received: 14 October 2020 / Revised: 9 November 2020 / Accepted: 17 November 2020 / Published online: 6 January 2021

(C) Springer-Verlag GmbH Germany, part of Springer Nature 2021

There is probably no congenital condition as horrid as intestinal malrotation. A seemingly normal child can present acutely, and, even if expediently diagnosed and operated on, might in a short time die or be left with severe lifelong debility from short gut syndrome. Getting the diagnosis quickly and correct is paramount.

A decade ago, we published two papers by Dr. David Yousefzedah on US for malrotation $[1,2]$. In Dr. Yousefzedah's hands (key point) and limiting the study to newborns, US performed well for differentiating normal from malrotation [2]. Dr. Yousefzedah looked for the third portion of the duodenum crossing midline posterior to the superior mesenteric artery. He demonstrated a retromesenteric course of the third portion of the duodenum in 33 of 33 newborns [2]. The papers received much attention. Many pediatric radiologists and groups have subsequently tried US for malrotation. Informally, we did in our practice. We failed. Two of my colleagues, both of whom I consider to be exceptionally talented with a transducer in hand, tried to emulate Dr. Yousefzedah's techniques but stated that they could not confidently exclude malrotation. If these two master sonographers could not, how could the rest of us?

Some practices, mostly outside the United States, have further pursued US for the diagnosis of malrotation [3-12]. Some have achieved substantial success. Most notable in the United States has been the group at Phoenix Children's Hospital [12]. In their hands, US was diagnostic in $92 \%$ of 195 patients, and, when diagnostic, both the negative and positive predictive values were $100 \%$ [12]. Other groups have published as well. A key, it would seem, for making the diagnosis of malrotation - or more critically (because this would stop further workup or treatment), for excluding the diagnosis of malrotation - is to be absolutely

Peter J. Strouse

Rad-Ped-Radiol-Journal@med.umich.edu

1 Section of Pediatric Radiology,

C. S. Mott Children's Hospital, Room 3-231, Department of Radiology, Michigan Medicine,

1540 E. Hospital Drive, Ann Arbor MI 48109-4252, USA certain that you are viewing the duodenum and not misidentifying another section of bowel as the retromesenteric portion of the duodenum. It is thus paramount to follow the bowel loop from the stomach and pylorus through its course behind the superior mesenteric artery.

Regardless of whether a practice chooses to use US to make the diagnosis of malrotation and volvulus, it is important to be cognizant of its appearance on US because the diagnosis might be present when not expected - a vomiting child from the emergency room; a child with chronic vomiting, abdominal pain or failure to thrive; a neonate with a distended abdomen who is clinically suspected of having necrotizing enterocolitis. In all of these instances, and others, one might encounter a malrotation with volvulus. If you don't know what a volvulus looks like, you might miss it or misinterpret it. If you don't look for a volvulus, you are likely to miss it.

In this issue of Pediatric Radiology, a group of authors from Texas Children's Hospital shares their experience with US for malrotation with volvulus. They have illustrated the US findings in an excellent pictorial essay [13]. They delineate their buy-in, training and ramp-up process in an accompanying commentary [14]. Texas Children's Hospital is the largest children's hospital in North America. It is very well resourced. The radiology group has around-the-clock in-house attending coverage in its main hospital. All US exams are performed by dedicated pediatric sonographers. The group does cover two smaller communitybased children's hospitals, which are included in this initiative, for which the US exams are interpreted remotely by in-house pediatric body radiologists at the main hospital. Based on the information provided, how are they doing? The results are good. But are they good enough? The authors noted that "Overall, ultrasound prospectively reported malrotation with midgut volvulus in 13 of 16 (81\%) [patients]" [14]. Even one missed volvulus needs be concerning. I, however, applaud the Texas Children's group for their willingness to test the waters, for their diligence in implementation and for carrying this through with relative success.

As evidenced by this work at Texas Children's Hospital, one must know the sonographic findings of volvulus and to 
specifically look for them. Everyone involved - sonographers, radiology residents and fellows and attendings - must have a strong working knowledge of the sonographic findings of malrotation and malrotation with volvulus. Any break in the chain - an untrained technologist, a resident starting call, a new attending who has not been trained bears a risk. You need to know what to look for. Training is continuous.

It is also crucial to know when to look. Patients don't always present with the classic bilious vomiting. Clinicians might not suspect the diagnosis. Whether formally involved in a protocol to evaluate for volvulus or not, it behooves one to be on the lookout.

A few years ago, we had a troublesome case. A 6-day-old infant presented to the emergency department (ED) in the middle of the night with "bilious vomiting." Without a physician having evaluated the child, the intake ED triage nurse mistakenly put in a request for a US exam to "rule out pyloric stenosis." The subsequent US was the most beautiful (if I am allowed to say so) example of malrotation and volvulus that I have seen on US - dilated duodenum coming to a beak with to-and-fro motion of contents going into a clockwise swirl of bowel and mesenteric vessels around the superior mesenteric artery in the central abdomen. The mesentery was edematous. (Too) many beautiful cines. What happened? A newly trained technologist took all the pictures but didn't recognize the diagnosis. A senior on-call resident viewed all of the images but didn't recognize the diagnosis. Together they reached other diagnoses, involving the surgeons, but not soon enough. Fortunately, one of my partners came in early that morning to get a head start on a busy day and prioritized this case first in a stack of overnight exams. A few seconds after opening the study, the diagnosis was clear. After directly calling the ED attending and a pediatric surgery attending, my colleague, shaken, came to our morning case conference and showed the case to the residents and fellows on service. I have since shown this same case dozens of times. Fortunately, in the operating room the child's dusky midgut pinked up; he survived and did not require any resection of necrotic gut. This story is illustrative of the crucial need for training and expertise in using US for malrotation with volvulus. Even if not using US in a formal protocol for detecting volvulus, one needs to know the findings. It is even more critical if you rely on US to make or exclude the diagnosis.

If a department is going to use US for malrotation and for volvulus, it requires resources to do so and to provide the training to do it at a high level of reliability. Our colleagues at Phoenix Children's Hospital and Texas Children's Hospital have strived for this. A strict protocol is required. Dedicated pediatric sonographers are required. Twenty-four/seven pediatric radiologist coverage is required. Unfortunately, this is not achievable by most pediatric radiology practices other than the largest.
One thing that bothers me about US for volvulus is that volvulus can be intermittent. Even if there is no frank volvulus at the instant that the US study is performed, how do I know that a child will not have a volvulus later tonight, tomorrow or next week? Where does the fine line between no volvulus and volvulus lie on US evaluation? Did the child not present with "bilious vomiting" that might have been caused by a volvulus, now temporarily resolved?

Do I want to come in at night and do several upper gastrointestinal (GI) series to rule out malrotation and volvulus for every one that is positive? Not really. But it is part of my job. It is rewarding to rule out such a potentially deleterious diagnosis. It is even more rewarding (after good news from the operating room) to save the life of a child by making the diagnosis and hurrying the child on her way toward curative surgery.

Some of the authors, with the help of others, have also published in another journal on the financial aspects of making the switch from upper GI series to US for volvulus [15]. Although it is interesting, I am leery of such an analysis. In regard to a diagnosis that is literally life-and-death, as much as anything that we do in pediatric radiology, it is accuracy that matters, not a few dollars here and there. The life of a child is priceless.

In regard to accuracy, it does no good to make comparisons between publications that negatively distort the accuracy of upper GI series for malrotation and malrotation with volvulus versus publications that positively distort the accuracy of US for malrotation and malrotation with volvulus. An upper GI series, though not perfect, is a good test. While we always look to minimize radiation exposure, the dose from a single upper GI series to make such a critical diagnosis should not be of concern, especially in the hands of a well-trained pediatric radiology fluoroscopist using modern fluoroscopy equipment. More study is needed that directly compares US to upper GI series. Unfortunately, such a study will require a large number of children to achieve necessary power to prove the superiority or non-inferiority of US, considering that one missed case is too many.

Again, I applaud the authors for their work, for their diligence and for their passion. At their institution, US for volvulus is working. Other institutions need to consider this carefully and, if committed, do it with equal dedication and care as done at Texas Children's Hospital.

One final thought. Has anyone tried MRI?

\section{References}

1. Yousefzadeh D (2009) The position of the duodenojejunal junction: the wrong horse to bet on in diagnosing or excluding malrotation. Pediatr Radiol 39:S172-S177 
2. Yousefzadeh D (2010) Assessment of retromesenteric position of the third portion of the duodenum: an US feasibility study in 33 newborns. Pediatr Radiol 40:1476-1484

3. Menten R, Reding R, Godding V et al (2012) Sonographic assessment of the retroperitoneal position of the third portion of the duodenum: an indicator of normal intestinal rotation. Pediatr Radiol 42: 941-945

4. Alehossein M, Abdi S, Pourgholami M et al (2012) Diagnostic accuracy of ultrasound in cause of bilious vomiting in neonates. Iran J Radiol 9:190-194

5. Khatami A, Mahdavi K, Karimi MA (2014) Ultrasound as a feasible method for the assessment of malrotation. Pol J Radiol 79:112116

6. Hennessey I, John R, Gent R, Goh DW (2014) Utility of sonographic assessment of the position of the third part of the duodenum using water instillation in intestinal malrotation: a single-center retrospective audit. Pediatr Radiol 44:387-391

7. Esposito F, Vitale V, Noviello D et al (2014) Ultrasonographic diagnosis of midgut volvulus with malrotation in children. J Pediatr Gastroenterol Nutr 59:786-788

8. Zhou L-Y, Li S-R, Wang W et al (2015) Usefulness of sonography in evaluating children suspected of malrotation: comparison with an upper gastrointestinal contrast study. J Ultrasound Med 34:18251832

9. Kumar B, Kumar M, Kumar P et al (2017) Color Doppler — an effective tool for diagnosing midgut volvulus with malrotation. Indian J Gastroenterol 36:27-31
10. Zhang W, Sun H, Luo F (2017) The efficiency of sonography in diagnosing volvulus in neonates with suspected intestinal malrotation. Medicine 96:42

11. Garcia AM, Asad I, Tessaro MO et al (2019) A multi-institutional case series with review of point-of-care ultrasound to diagnose malrotation and midgut volvulus in the pediatric emergency department. Pediatr Emerg Care 35:443-447

12. Wong K, Van Tassel D, Lee J et al (2020) Making the diagnosis of midgut volvulus: limited abdominal US has changed our clinical practice. J Pediatr Surg. https://doi.org/10.1016/j.jpedsurg.2020.04. 012

13. Nguyen HN, Navarro OM, Guillerman RP et al (2020) Untwisting the complexity of midgut malrotation and volvulus ultrasound. Pediatr Radiol. https://doi.org/10.1007/s00247-020-04876-x

14. Nguyen HN, Sammer MBK, Ditzler MG et al (2020) Transition to ultrasound as the first-line imaging modality for midgut volvulus: keys to a successful roll-out. Pediatr Radiol. https://doi.org/10. 1007/s00247-020-04913-9

15. Nguyen NH, Sammer MB, Bales B et al (2020) Time-driven activity-based cost comparison of fluoroscopic upper gastrointestinal examination versus focused abdominal ultrasound for suspected midgut volvulus in children. J Am Coll Radiol. https://doi.org/10. 1016/j.jacr.2020.06.023

Publisher's note Springer Nature remains neutral with regard to jurisdictional claims in published maps and institutional affiliations. 